\title{
SNAPSHOT
}

\section{Mapping photosynthesis}

Researchers have created a global map of the fluorescence emitted by landbased plants during photosynthesis. This subtle glow at certain wavelengths could serve as an early warning system for plant stress and help scientists better understand Earth's carbon cycle.

During photosynthesis, plants use chlorophyll and solar energy to convert carbon dioxide, water and other nutrients into sugars and other organic compounds. Together, these chemical reactions emit low levels of fluorescent light at wavelengths between $650 \mathrm{~nm}$ (red) and $800 \mathrm{~nm}$ (near-infrared). But this feeble glow typically goes unnoticed because it is swamped by sunlight reflected from the plant, says Joanna Joiner, a remote-sensing analyst at NASA's Goddard Space Flight Center in Greenbelt, Maryland. However, at some wavelengths - particularly the ones at which most or all of the radiation is absorbed by ionized atoms in the Sun's outer atmosphere - the fluorescence shines forth unmasked. And lab tests suggest that when sunlight is bright, the strength of fluorescence is a good indicator of how much photosynthesis is taking place.

Using data gathered by a Japanese satellite originally designed to observe atmospheric concentrations of greenhouse

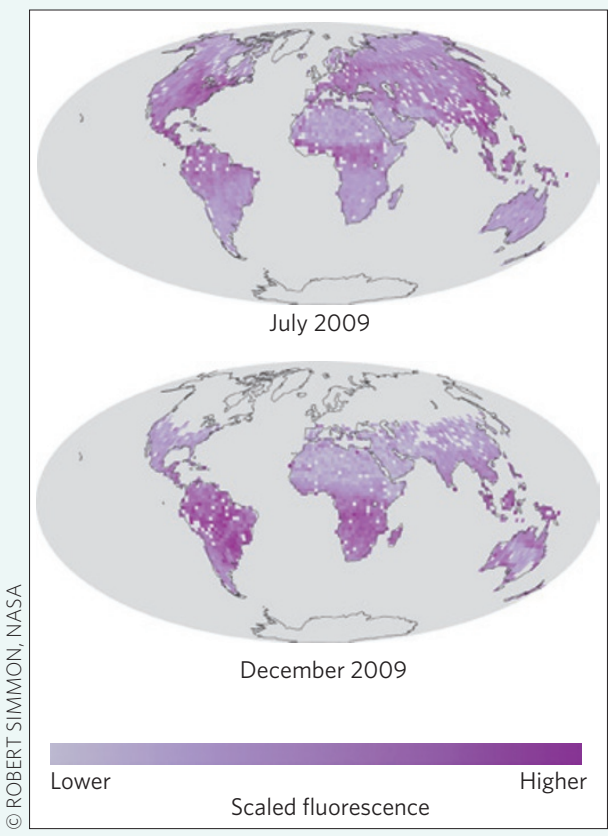

gases worldwide, Joiner and her colleagues mapped the fluorescence emitted in 2009 by land-based vegetation at $770 \mathrm{~nm}$ (Biogeosciences 8, 637-651; 2011), a wavelength usually stripped from sunlight by potassium ions. The team's analyses reveal that, as expected, levels of plant fluorescence vary with the seasons, peaking during July in the Northern Hemisphere and in December below the Equator

Current satellite-based schemes to assess the health of vegetation depend on measurements of plant 'greenness', which typically wanes in the wake of droughts, frosts or other sources of stress. However, visible changes in leaf colour sometimes take days or even weeks to develop, so fluorescence can provide a more direct and more timely insight into plant stress, says Joiner. The team suggests that because anything affecting photosynthetic activity will in turn influence fluorescence, this new method might be able to detect plant stress due to other factors as well, including ground-level ozone or other pollutants.

Besides offering an early warning system for plant health, the fluorescencebased technique could help international aid workers detect and, perhaps, predict famines, thereby decreasing the time needed to mount an effective response. Moreover, the researchers say, monitoring long-term trends in plant fluorescence could help scientists improve their understanding of how carbon is cycled through Earth's ecosystems - one of the key areas of uncertainty in climate science.

\section{SID PERKINS}

\section{Facing the heat}

\author{
Marc Hudson
}

\section{Scientists discuss the consequences of allowing global carbon emissions to continue unmitigated - and describe a scary future.}

n Nevil Shute's 1957 classic Australian novel $O n$ The Beach, a bewildered but stoic group of people in Melbourne wait out the end of their world in the aftermath of a global nuclear war. Half a century later, delegates gathered in Melbourne to discuss another imminent threat to human civilization - but this time it's a non-fictional possibility. The three-day 'Four Degrees Or More?' conference held in July this year drew climate scientists from around the world to discuss the implications of average global temperatures increasing by four degrees above pre-industrial levels - a likely scenario by 2100 , if not decades sooner, under current carbon emission trajectories.

The outlook for Australia would be brutal, the scientists reported: agricultural production severely slashed by mid-century, forcing a reliance on imports; sea levels rising more than one metre; record high temperatures (Table 1) and extreme fires; 